\title{
OBSERVATIONS RELEVANT TO THE IMPROVEMENT OF THE FUNDAMENTAL SYSTEM
}

\author{
W. GLIESE \\ Astronomisches Rechen-Institut, Heidelberg, F.R. Germany
}

\begin{abstract}
Since 1950 about 150 observational catalogues have become available which may contribute to the improvement and extension of the FK4. Twenty-five of these have been classfied as 'absolute' or 'quasi-absolute' by their authors. Part of the observations, however, are not yet available in publications or in a form suited for data processing. Some of the absolute observations in R.A. suffer from insufficient determination of the azimuth and others in Decl. from unclear elimination of flexure. Main deficiencies in the system of the FK4 are inaccurate positions and proper motions in right ascensions south of $-35^{\circ}$.
\end{abstract}

A search for observations relevant to an improvement and extension of the fundamental catalogue yielded about 150 catalogues published after 1950 which have not been included in the FK4. About twenty more catalogues have been observed which are not yet published.

Most of the positions have been observed differentially and will allow an improvement of the individual positions and proper motions.

Only about thirty catalogues will contribute also to an improvement of the fundamental system of positions and proper motions.

Such observations are called 'absolute' even if they do not include the determination of the equator and the equinox. They should fulfil two conditions:

(1) The observed positions have been determined without any reference to stellar positions in a basic catalogue, or, if such basic positions have been used, their systematic effects must have been eliminated by a suitable reduction.

(2) The effects of instrumental errors on the observed positions must be eliminated.

The first condition is often fulfilled only for parts of the observed data. The second condition will never be fulfilled exactly but only approximately.

Different observational methods have been employed and the behaviour of instruments varies from catalogue to catalogue. Therefore, data classified as 'absolute' by their authors will contribute with different weights to a fundamental system but there are no strong rules for computing such weights.

The compilers of a fundamental system need detailed information on methods of observations and on instrumental behaviour during the observational periods. Introductions and texts to observed catalogues sometimes describe the methods of observation and reduction in insufficient detail; in some cases, questions concerning old catalogues will never be answered.

Special attention should be given to the determination of the azimuth in observations of absolute right ascension. In declination, the elimination of flexure errors will influence significantly the weights of absolute observations. Preliminary reviews on possible errors in the FK4 system have been given at Prague (Fricke and Gliese, 1968) and at Minneapolis (Gliese, 1970). The declination system seems to be virtually 
correct. The large $\Delta \alpha_{\delta}$ error in southern declinations is the weakest point in the FK4. Dr Anguita will present a detailed report on this situation later in this symposium. I should emphasize that, from the new observational series in the southern hemisphere, a reliable fundamental right ascension system at epoch about 1968 will be derived in a few years.

However, most astronomers are much more interested in a fundamental proper motion system free from errors than in positions accurate just for one special epoch.

It seems to me very difficult to obtain first epoch absolute right ascension catalogues relevant to the derivation of a good proper motion system in the south. Neither FK3 nor N30 nor FK4 have yielded such a system. Their southern systems have been based mainly on the catalogue series observed with Gill's Transit Circle at the Cape. In spite of all careful work of the observers, unexplained variations of the instrumental system occurred during its fifty years of action. Some at least of these catalogues, for example the $1 . \mathrm{Cp}_{50}$, should not be used for the compilation of a fundamental right ascension system.

On the other hand, it is very interesting to notice that an intensive but short series observed during the Cape winter 1936 already shows the $\Delta \alpha_{\delta}$ errors in the fundamental systems. The special value of this small catalogue lies in the very accurate determination of the azimuth by many successive culminations observed from April to September.

We have to look once more very carefully into the work done at the Cape Observatory in the first decades of this century, but I have some doubt whether the observations at Cordoba and at Melbourne can contribute significantly to this problem.

Therefore, although I expect a fundamental system remarkably improved especially in the southern hemisphere in the near future, I am sceptical about any improvement in the right ascension proper motion system south of $-45^{\circ}$.

\section{References}

Fricke, W. and Gliese, W.: 1968, in L. Perek (ed.), Highlights of Astronomy, D. Reidel Publ. Co., DordrechtHolland, p. 301.

Gliese, W.: 1970, in W. J. Luyten (ed.), 'Proper Motions', IAU Colloq. 7, 146.

\section{DISCUSSION}

Stoy: I think that the odd behaviour of the southern FK4 right ascension system is to be traced back to the different methods used for observing the slow moving polar stars which has resulted in an apparent twist of the polar cap relative to the rest of the sky. Once such a twist was in the system it was perpetuated by the practice since the publication of FK 3 of adopting the FK3 places for both the clock and azimuth stars. The situation has been further complicated by the use of a different selection of azimuth stars for different catalogues, but, now it is known that this effect is present, it should be possible to trace it through the various catalogues and correct for it.

Gliese: I agree with you that a re-investigation of former Cape catalogues based on improved positions of the azimuth stars will result in corrected catalogue systems but not necessarily in independent 'absolute' systems. In any case, an enormous amount of work would be needed. 